\title{
Discrete event based hybrid framework for petroleum products pipeline activities classification
}

\author{
S. S. Udoh ${ }^{1}$, O. C. Akinyokun*2, U. G. Inyang ${ }^{1}$, O. Olabode², G. B. Iwasokun² \\ ${ }^{1}$ Department of Computer Science, Faculty of Science, University of Uyo, Uyo, Nigeria \\ ${ }^{2}$ Department of Computer Science, Faculty of Sciences, Federal University of Technology, Akure, Nigeria
}

Received: May 2, 2016

Accepted: June 19, 2016

Online Published: April 6, 2017

DOI: $10.5430 / a i r . v 6 n 2 p 39$

URL: https://doi.org/10.5430/air.v6n2p39

\begin{abstract}
The importance of timely detection, classification and response to anomalies on petroleum products pipeline (PPP) have attracted pragmatic researches in recent times. There is need for efficient monitoring and detection of activities on PPP to guide leak detections and remedy decisions. This paper develops an intelligent hybrid system, driven by discrete event system specification (DEVS) and adaptive neuro-fuzzy inference system (ANFIS) for detection and classification of activities on PPP. A dataset comprising 330 records was used for training, validation and testing of the system. Result of sensitivity test shows that inlet pressure, inlet temperature, inlet volume and outlet volume have cumulative significance of $71.72 \%$ on flowrate of PPP. Hybrid learning algorithm was observed to converge faster than the back propagation algorithm in the detection of pipeline activities. ANFIS hybrid learning algorithm with training and testing errors of 0.11980 and 0.010233 yielded a correlation of 0.916 between the computed and the desired output and produced optimal consequent parameters to boost the intelligence of DEVS. A testing error of 0.0303 was observed in the evaluation of DEVS-ANFIS system on 33 test data sample, 32 precise detections were made with one incorrect detection, this gives $96.97 \%$ level of confidence in the DEVS-ANFIS model for detection, classification and localization of PPP activities.
\end{abstract}

Key Words: Discrete event system specification, Adaptive neuro-fuzzy inference system, Hybrid learning, Oil pipeline, Intelligent DEVS

\section{INTRODUCTION}

Over the years, petroleum products have sustained its role as a major and dependable source of fuel and income in many economies of the world. The transportation, distribution and delivery of crude oil and its products is a large-scale global business and an important component of the petroleum industry downstream sector. It focuses on the movement of natural gas, crude oil and other refined petroleum products from the refining countries to the consuming countries as well as distribution and sales of such products within a country or region. ${ }^{[1,2]}$ This can be carried out through trucking, railroad tank-cars, water carriers and pipelines. The choice of any of these modes of transportation depends on the cost. ${ }^{[3]}$ While trucks are the most expensive, the cost of rail remains multiples of pipeline and marine options. Pipeline is the cheapest and safest medium, hence a widely used mode of transportation and distribution of crude oil and its products. They are, therefore, the irreplaceable core of the petroleum products transportation system and the key to meeting petroleum products demand in the world. They provide transportation, temporary storage and logistics services in the oil and gas industries. ${ }^{[3,4]}$

\footnotetext{
${ }^{*}$ Correspondence: O. C. Akinyokun; Email: admin@akinyokun.com; Address: Department of Computer Science, Faculty of Sciences, Federal University of Technology, Akure, Nigeria.
} 
The unavailaibility of appropriate guidelines, policies and standards for the design, construction, and operation of oil pipelines in many developing countries has affected the quality, durability and integrity of pipelines. ${ }^{[2]}$ Causes of pipline failure include structural problems $(40 \%)$, operator errors $(6 \%)$, external influence damage (27\%), control problems (2\%) and others (25\%). Besides petroleum products pipeline (PPP) failure, the tampering with PPP and other oil installations, which has assumed wide dimensions through any of these forms; oil bunkering, oil theft, fuel scooping, militancy (oil terrorism) and PPP vandalism is another source of oil spillage from PPP. PPP vandalism is the deliberate destruction of PPP with the intent of stealing petroleum products or sabotaging government politically or economically. ${ }^{[5]}$

The risk associated with PPP failure and vandalism in terms of safety of people(security), damage to the ecosystem, loss of income, threat to infrastructural and economic development, has been a central issue to PPP integrity managers, host communities, oil prospecting companies, governments and other stakeholders. ${ }^{[6]}$ In many developing economies, there are no contingency plans for rapid response to oil spillages, poor detection and mitigation procedures, which in turn increases the risks associated with PPP failures and vandalism. In consideration of these negative impacts, there is need to effectively monitor, protect and prevent activities with negative effect on pretroleum products transportation. These activities on PPP domain could be classified as normal, abnormal, highly abnormal or extremely abnormal in response to varying and dynamic attributes like pressure, flowrate, temperature, viscosity, density and other PPP parameters. The impact of petroleum product spillage could be mitigated by an effective PPP monitoring system. ${ }^{[7]}$ PPP monitoring involves constant checks on events of oil spillage-induced parameters with a view to providing real time information and response to events with significant deviation from the stipulated threshold of normal operations. Regular monitoring with parameters featuring the structural and functional conditions of the flow line can help prevent failure, detect a problem and its position and undertake maintenance and repair activities in time. ${ }^{[7]}$ Different approaches have been used to evolve systems that monitor, detect, classify or respond to emergencies resulting from oil spillages and leakages. ${ }^{[8-15]}$ These systems are limited by lack of a systematic way of tracking the time of activities, high probability of false detection and inefficient localization of detected activities due to non inclusion of intelligent tools for explicit timing of operations, pattern recognition and data imprecision handling. Discrete event system specification (DEVS) offers a plausible solution for the specification of timing and localization need of this problem, while adaptive neuro-fuzzy inference system (ANFIS) proffers solution for pattern recognition and data imprecision. DEVS is a set-theoretic mathematical formalism for structural representation and organisation of similar or diverse computing tools in solving time-relevant and complex problems. ${ }^{[16,17]}$ DEVS has been successfully applied in wild fire spread and containment $;^{[18]}$ sensing and detection of intruder in a network environment; ${ }^{[19]}$ wind speed detection; ${ }^{[20]}$ environmental fire detection; $;^{[21]}$ monitoring of oil pipelines. ${ }^{[22]}$ DEVS has a formal means for explicit specification of timing operations, which is central for real-time systems. ${ }^{[23,24]}$ In DEVS, the state and state transitions of the source system are dynamic, in response to occurrences of events which are inherently imprecise, uncertain and vague. ${ }^{[25]}$ DEVS systems are good at specifying timing of events but lack the cognitive ability to recognize patterns or handle imprecise data. ${ }^{[17]}$ The combination of DEVS with intelligent tools that recognise patterns and process imprecise data into a single system becomes one of the best approaches for classification of activities on PPP. These intelligent tools include neural networks (NN), fuzzy logic (FL), and genetic algorithm (GA) and so on.

This paper proposes a framework that integrates ANFIS into DEVS where the unique strengths of each sub-system will be enhanced and the weaknesses compensated in the detection and classification of activities on PPP. The remainder of this paper is organized as follows. Section 2 presents a review of related literature while the methodology, data collection and system design techniques of ANFIS and DEVS for pipeline activities classification are carried out in Section 3. Section 4 gives the implementation techniques and discussion of output results. The conclusions of the paper and recommendations for further research are presented in Section 5.

\section{RELATED WORKS}

In Filippi and Bisgambiglia, ${ }^{[26]}$ DEVS-NN model is proposed for detection of atmosperic gas in a plot of land. The DEVS-NN hybrid provides intelligent system timing operations and automatic coordination of the system learning capabilities but lacks the ability to handle imprecise and vague information. In Prasanna et al. ${ }^{[19]}$ DEVS-FL model is proposed to address imprecision in the detection of intruders in a networked environment. The DEVS-FL system established a platform for keeping time of attacks on computer networks as well as automatic coordination of membership function computation, inferencing and defuzzification procedures but lacks the facility for collective situational awareness and training. In Niklès et al. ${ }^{[8]}$ a fibre optics system is presented to monitor, detect and localize defects in PPP. The fibre optics system provided real-time information about the pipeline integrity which guided management decision but was charac- 
terized by high frequency of false detection and localization of oil spills due to non inclusion of intelligent tools to aid decision in human-like pattern. In Daniel and Mihaela, ${ }^{[9]}$ an intelligent system for monitoring pipeline leakages using FL technology is proposed. The FL system facilitated humanlike classification of oil leakages in pipeline infrastructure but cannot learn from previous input variables and generalization into unseen patterns. Motivated by the need to overcome the low success rates recorded by traditional and mechanical systems in oil spill emergency response, FL system to monitor, detect, localize and respond to oil spill emergency situations in Artic waters was proposed. ${ }^{[27]}$ Though the system could capture, store and encapsulate substantial knowledge pertaining to location and severity of oil spillages, vague and imprecise timing related parameters of oil spill events in the marine environment were not formalized and modelled appropriately, thereby hindering efficient rescue decisions. Lin and Ying ${ }^{[28]}$ extended crisp DEVS system by incorporating fuzzy set theory (fuzzy finite automaton model) into DEVS. In Santucci and Capocchi, ${ }^{[29]}$ the integration of FL into DEVS spans three steps; definition and characterization of FL concepts of the problem domain (fuzzification, fuzzy rules definition and firing as well as defuzzification). The next stage focuses on choosing the programming language environment for the integration of FL and DEVS. In the final stage, the implementation and integration of the design into DEVS framework is proposed. A methodology for the enhancement of DEVS formalism through the integration of cognitive reasoning capabilities into the classical DEVS system is proposed in Sheikh-Bahaei et al. ${ }^{[30]}$ The results obtained showed a better performance than traditional algorithms, especially when implemented with a large number of rules and membership functions.

In Udoh, ${ }^{[10]}$ the practical value of $\mathrm{NN}$ is demonstrated in a petroleum products distribution depot. The NN technique enabled system training and generalization into future patterns but lacks ability to deal with imprecise and vague petroleum products data. In Adewuyi and Okelola, ${ }^{[13]}$ pipeline leak detection and control is presented using NN. The NN tools produced an intelligent system with learning capabilities for detecting the anomalies on PPP but were not capable of dealing with imprecise PPP data. In Akinyokun and Inyang, ${ }^{[12,14]}$ oil spillage risk managment framework is proposed using neurofuzzy-genetic platform. The neuro-fuzzy-genetic system demonstrated optimized training and imprecise data handling capabilities in the task of recognising patterns in complex oil spillage dataset but lacks facility for timing and automatic management of simultaneous oil spillage-induced parameters from multi pipeline locations. A pattern discovery system is also developed from NN training based on modified Apriori

Published by Sciedu Press rule-mining technique. The system enhances learning from previous data, identification, extraction and classification of oil spillage patterns but lacks the capability for timing and automatic monitoring of events that led to the oil spillage on PPP. In Santos et al. ${ }^{[11]}$ prediction of leak magnitude in a gas pipeline is presented using NN and acoustic sensors. The NN and acoustic sensors hybrid produced accurate predictions for high frequency signals of oil leakages but could not give correct predictions for low frequency signals even under occurrences of leakages. In Goran et al.,${ }^{[31]}$ evacuation model at liquefied natural gas (LNG) terminal is proposed using FL techniques. The FL system minimises the possibility of errors during the selection of an evacuation route in accident situations but lacks components for learning from previous accidents and route evacuation patterns.

An ANFIS-based scheme for petroleum pipeline monitoring by training a set of natural frequencies and time-domain signals from acoustic emission sensors is proposed in Vishash. ${ }^{[7]}$ The signals were generated by simulating leakage and corrosion in the pipeline and by varying the pressure and flow rate. The system demonstrated the ability of ANFIS-based system in learning from previous petroleum pipeline data, dealing with imprecise knowledge and making correct predictions on the test data but lacked the capability of timing of oil pipeline events as well as dealing with simultaneous events from multi-pipeline locations. In Mashford et al., ${ }^{[32]}$ an approach for monitoring leakages in pipeline infrastructure using support vector machine (SVM) is presented. The system lacked the capability for automatic control and timing of pipeline leakages to guide effective rescue operations.

The limitations in the existing works could be surmounted by integrating DEVS and ANFIS into a single system where their unique strengths are harnessed and their weaknesses eliminated in the task of monitoring PPP.

\subsection{Classical and fuzzy DEVS atomic models descrip- tion}

A Classical DEVS is expressed as a set of rules or mathematical equations used to approximate the inputs and outputs trajectories of the source systems. ${ }^{[23]}$ The basic formalism called Atomic Model (AM) in Zeigler ${ }^{[33,34]}$ is represented as follows:

$$
\mathrm{AM}=\left\langle X, Y, S, \delta_{\text {ext }}, \delta_{\text {int }}, \lambda, t_{a}\right\rangle
$$

where $X$ is a set of external inputs events, $Y$ is the set of external output events, $S$ is a set of states, $\delta_{\text {ext }}$ : $Q \times X \rightarrow S$ is the external transition function where $Q=\left\{(s, e) \mid s \in S\right.$ and $\left.0 \leq e \leq t_{a}(s)\right\}$; is the total state set, $e$ is the time elapsed since last transition. $\delta_{i n t}: S \rightarrow S$, 
is the internal transition function; $\lambda: S \rightarrow Y$, is the output function; $t_{a}: S \rightarrow R^{+}{ }_{0, \infty}$, is time advance function.

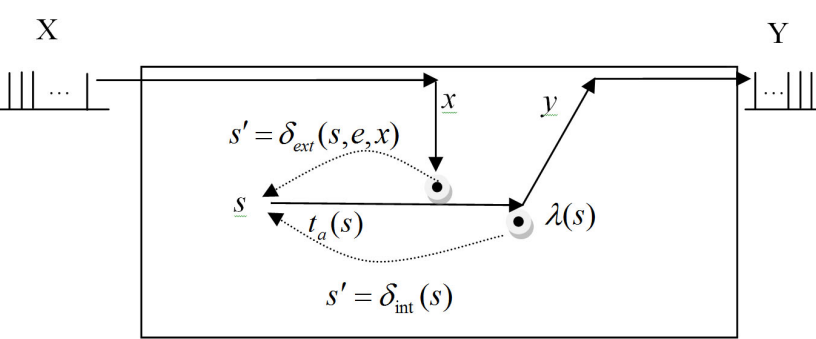

Figure 1. Elements of DEVS structure ${ }^{[33]}$

The interaction of these elements is depicted in Figure 1.

The AM model of DEVS is extended in Chow and Zeigler. ${ }^{[35]}$ A proposal of Parallel DEVS (PDEVS) formalism is presented with the incorporation of confluent function into the classical DEVS to facilitate parallel processing. The PDEVS model is given as:

$$
\mathrm{PDEVS}=\left\langle X, Y, S, \delta_{\text {ext }}, \delta_{\text {int }}, \delta_{\text {con }}, \lambda, t_{a}\right\rangle
$$

where $X, Y, S, \delta_{\text {int }}, \delta_{\text {ext }}, \lambda$ and $t_{a}$ are the same as classical DEVS model. $\delta_{\text {con }}: Q \times X^{b} \rightarrow S$ is the confluent transition function, $b$ is a collection of input events.

In Fuzzy-DEVS hybridization, a human like reasoning capabilities of FL are exploited to process imprecise or vague data while DEVS handles timing related issues and automatic system control. ${ }^{[36]}$ The Fuzzy-DEVS model involves the following Membership Functions (MF) atomic models, antecedent MF (AMF) - Equation (3), Consequent MF (CMF)- Equation (4), Defuzzfiers MF (DMF) - Equation (5), and connectives Equation (6). ${ }^{[30]}$

$$
\begin{aligned}
& \mathrm{AMF}=\left\langle X, Y, S, \delta_{e x t}, \delta_{i n t}, \delta_{c o n}, \lambda, t_{a}, \mu_{a}\right\rangle \\
& \mathrm{CMF}=\left\langle X, Y, S, \delta_{\text {ext }}, \delta_{\text {int }}, \delta_{\text {con }}, \lambda, t_{a}, \mu_{c}\right\rangle
\end{aligned}
$$

$$
\mathrm{DMF}=\left\langle X_{1}, X_{2}, \cdots, X_{n}, Y, S, \delta_{\text {ext }}, \delta_{\text {int }}, \delta_{\text {con }}, \lambda, t_{a}\right\rangle
$$

$$
\mathrm{CNT}=\left\langle X_{1}, X_{2}, \cdots, X_{n}, Y, S, \delta_{e x t}, \delta_{i n t}, \delta_{c o n}, \lambda, t_{a}, \mu_{d}\right\rangle
$$

where $X$ is a set of real values that represents the inputs. $Y=[0,1]$ the output set, $S$ is the fuzzified input sequence $S=\left\{s_{i} \mid s_{i}=\delta_{\text {ext }}\left(q, s_{i-1}, x\right)-\delta_{\text {ext }}(q, s, x)\right\}=\mu(x)$, where $\mu(x)$ is the MF associated with this fuzzifier. $\delta_{\text {int }}(s)$ and $\delta_{c o n}(s)$ are the identity functions. $t_{a}(s)=0$ denotes the time advance.

A consequent MF defined in Equation 4 has the input values, $X=[0,1]$, while the output $Y$ is a fuzzy set. $Y=\{\Sigma \mid \Sigma$ is a fuzzy set $\}, S$ is a sequence of MF values $S=\cdots, \Sigma_{i-1}, \Sigma_{i}, \Sigma_{i+1}, \cdots, \mu(x)$ is the MF associated with this fuzzifier.

$$
\delta_{\text {ext }}(q, s, x)=\sum \frac{\min \left(\mu\left(a_{i}\right), x\right)}{a_{i}}
$$

The connectives - Equation (6) provides link between antecedent and consequent pairs using AND/OR operators. An AND connective takes the minimum, while an OR returns the maximum value of two pairs. ${ }^{[37]}$ A description of the AND and OR is provided in Equations (8) and (9). ${ }^{[30]}$

$$
\begin{aligned}
& \delta_{\text {ext }}\left(q, s, x_{1}, \cdots, x_{n}\right)=\min \left(x_{1}, \cdots, x_{n}\right) \\
& \delta_{\text {ext }}\left(q, s, x_{1}, \cdots, x_{n}\right)=\max \left(x_{1}, \cdots, x_{n}\right)
\end{aligned}
$$

A fuzzy rule is a coupled model which consists of AMF, CMF, CNT and DFM atomic models. The AMF are activated when new inputs events are received, it generates output events when the new fuzzified values are not the same with the earlier ones. In addition, CNT models are activated only when there is a variation in their inputs values. Tunning can be achieved either by adding extra input to the DEVS models or modifying the parameter simplicitly. $\mathrm{NN}$ is a suitable tool and widely adopted in both cases.

\section{RESEARCH METHODOLOGY}

The stages of the research, presented in Figure 2, begin with dataset collection and pre-processing followed by the DEVSANFIS design and implementation. A dataset of 330 data sample was collected from Pipeline and Products Marketing Company (PPMC) in Port Harcourt Area Office, Nigeria, covering Port Harcout and Aba (PHAB), Port Harcourt and Enugu (PHEN) as well as Enugu and Markurdi (ENMK) pipeline segments. The dataset was divided into training, validation and testing datasets in the ratio of 8:1:1 which translates into 264 records for training and 33 records each for validation and testing. Fourteen (14) input indicators and one (1) output variable describing PPP behavior were captured in the dataset.

The next stage was the pre-processing and dimension reduction using Neuro Solutions. The attribute description and dimension reduction results are presented in Table 1. Variables IPS, IVS, ITP, PDM, PLT, CRN, TPS, OVS, OTP, OPS, 
IVM, DTY, VSC, and OVM are inputs attributes, while the FLR is the output variable. The attribute contribution to the FLR shows that IPS has the highest score of $65.32 \%$, while IVM and OVM have a score of $55.10 \%$ and $50.19 \%$ respectively. The variables ITP, VSC, TPS, OTP, DTY and OPS scored above $10 \%$ each. PLT and PDM scored $4.23 \%$ and $3.00 \%$ respectively while IVS and OVS contibuted $0.12 \%$ each to the determination of output. The best four scorers are IPS, IVM, OVM and ITP. Hence these best performing attributes are used as inputs to the system, pruning the others.

Table 1. Sensitivity test of PPP attributes to flow rate

\begin{tabular}{lllll}
\hline SN & Attributes & Sensitivity & $\begin{array}{l}\text { Score } \\
\text { (\% ) }\end{array}$ \\
\cline { 2 - 3 } & Description & Code & & 65.32 \\
2 & Inlet_Pressure & IPS & 0.653155 & 55.1 \\
3 & Inlet_Volume & IVM & 0.551048 & 50.19 \\
4 & Outlet_Volume & OVM & 0.501936 & 50.75 \\
5 & Inlet_Temperature & ITP & 0.307525 & 30.75 \\
6 & Viscosity & VSC & 0.212619 & 21.26 \\
7 & Transient_Pressure & TPS & 0.149851 & 14.99 \\
8 & Outlet_Temperature & OTP & 0.119292 & 11.93 \\
9 & Density & DTY & 0.115519 & 11.55 \\
10 & Outlet_Pressure & OPS & 0.10982 & 10.98 \\
11 & Pipe_Length & PLT & 0.042343 & 4.23 \\
12 & Corrosion & PDM & 0.030021 & 3.00 \\
13 & Outlet_Valve_Size & ORN & 0.012341 & 1.23 \\
14 & Inlet_Valve_Size & IVS & $1.23 E-03$ & 0.12 \\
\hline
\end{tabular}

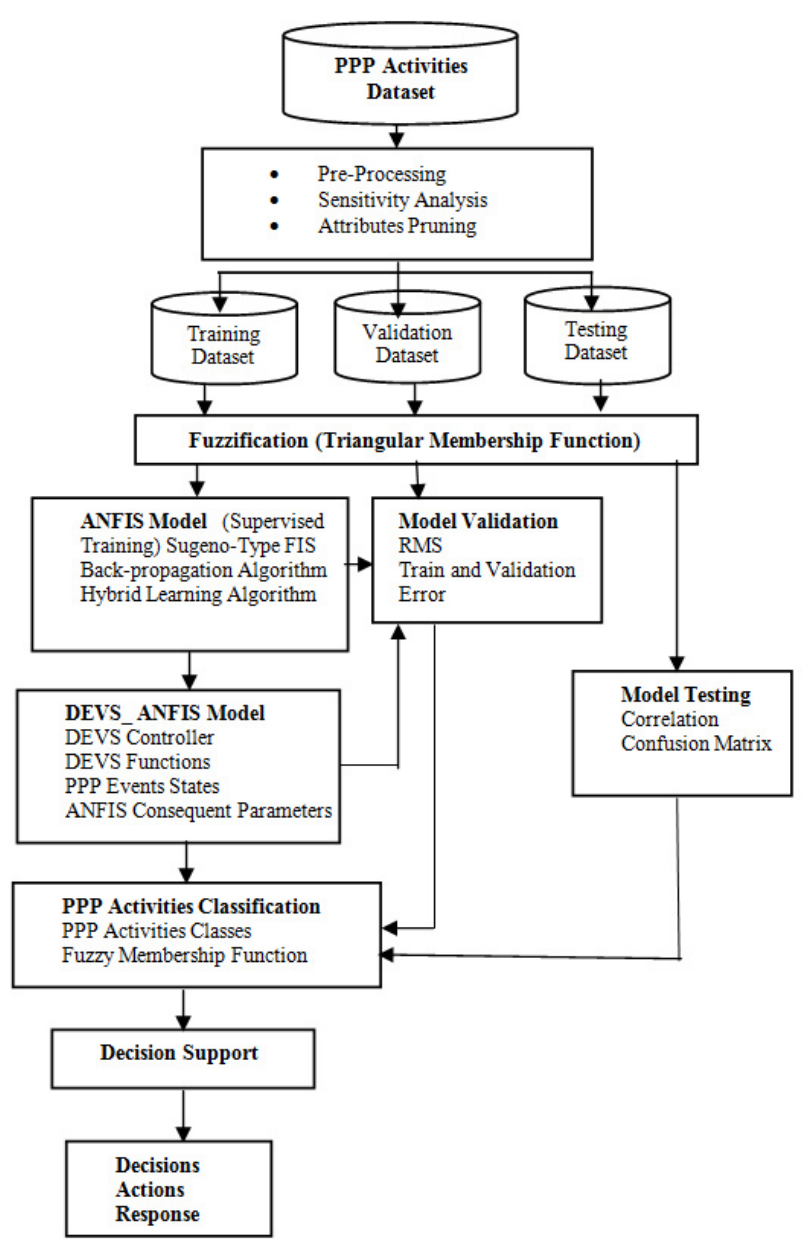

Figure 2. DEVS-ANFIS development stages

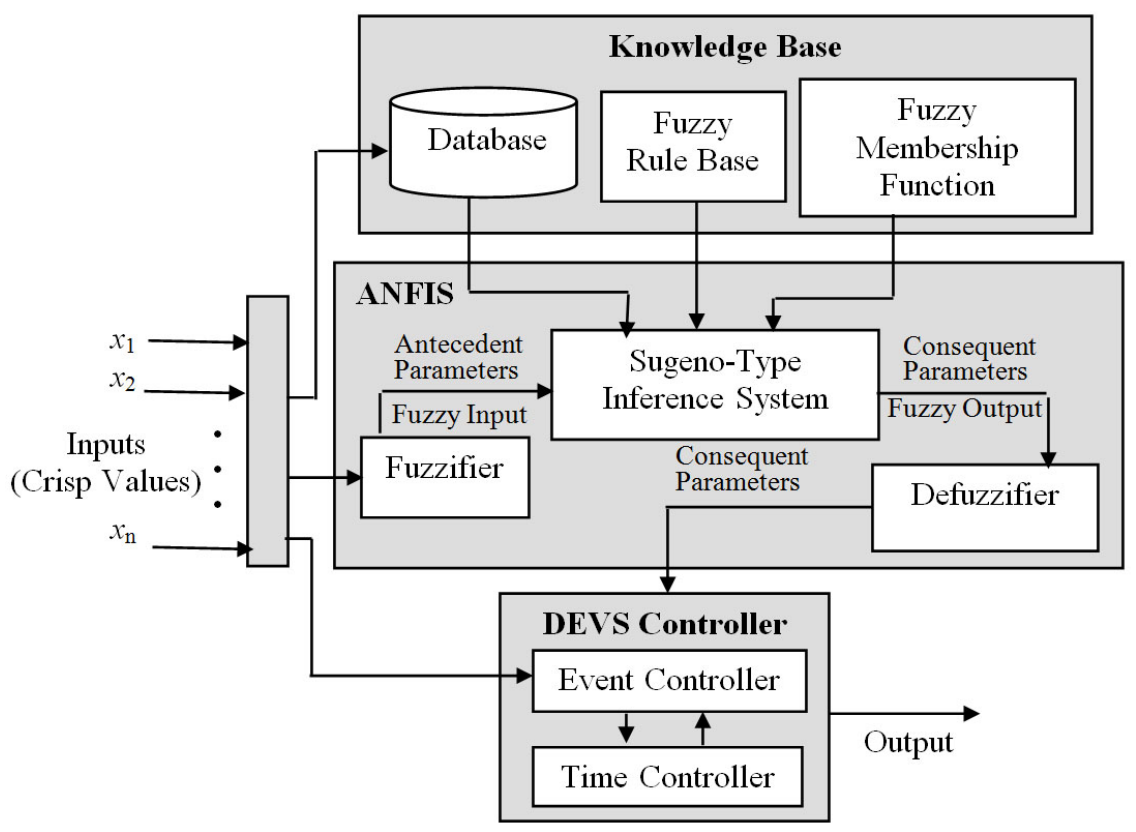

Figure 3. Block diagram of DEVS-ANFIS system 
The block diagram of the system (see Figure 3 ) consists of three parts; Knowledge Base (KB), ANFIS and the DEVSController. The KB contains fuzzy Membership Functions (MF), fuzzy rulebase and the database. The Database represents the properties of fuzzy sets, rules and membership function. The ANFIS consists of fuzzifier, Sugeno inference engine and defuzzfier while the DEVS controller has in addition to it atomic components, the Events and the Time controllers.

\subsection{ANFIS}

The ANFIS is designed for descrete events parameter identification through a hybrid learning rule combining the backpropagation gradient descent and a least-squares method. ANFIS is basically a graphical network bestowed with the neural learning capabilities. The network comprises adaptive and fixed nodes with specific functions arranged in five (5) layers. The input layer consists of PPP attributes. The system is based on Sugeno inference mechanism whose reasoning methodology presents the output of each rule as a sequential combination of each rule input variable plus the constant term as shown in Equation (10).

IF $x$ is $A, y$ is $B$ and $z$ is $C$, then

$$
f=p x+q y+\cdots+r z+s
$$

where $x, y, z$ are the inputs or antecedent parameters; $A$, $B, C$ are the fuzzy sets of inputs parameters, $f$ is the fuzzy set of output parameters and $p, q, r$ and $s$ are consequent parameters.

The fuzzifieris in layer one, accepts crisp values and transforms them into linguistic variables. The membership functions defined on the input values are applied to their actual values in order to determine the degree at which they belong to a particular fuzzy set. Every node $i$ in layer 1 has a node function:

$$
O_{i}^{1}=\mu A_{i}(x)
$$

where $x$ is the input to node $i$, and $A_{i}$ is the linguistic label (Very Low, Low, Moderate, High and Very High) associated with this node function. $O_{i}^{1}$ is the MF of $A_{i}$ and it specifies the degree to which the given $x$ satisfies the quantifier $A_{i}$. It is confirmed in Inyang and Akinyokun ${ }^{[14]}$ that the triangular MF performs better than others in the task of predicting and classifying oil spillage patterns. The general form of a triangular MF is shown in Equation (5).

$$
\mu_{A}(x)=\left\{\begin{array}{cc}
1 & \text { if } x=b \\
\frac{x-a}{b-a} & \text { if } a \leq x<b \\
\frac{c-x}{c-b} & \text { if } b \leq x<c \\
0 & \text { if } c=x
\end{array}\right.
$$

where $a, b, c$ are the parameters of the MF governing triangular shape such that $a \leq x<b, x$ is the external input from PPP. Layer 2 computes the firing strength of each rule as given in Equation (13), while nodes in layer 3 calculate the ratio of the $i$ th, rule's firing strength to the sum of all rules's firing strengths as shown in Equation (14). The defuzzifier (layer 4), which consists of consequent nodes for computing each rule contribution to the overall output is given in Equation (15). Layer 5 has a single node and it computes the overall output as the summation of all incoming signals using Equation (16).

$$
\begin{aligned}
& O_{i}^{2}=w_{i}=\mu A_{i}(x) \times \mu B_{i}(y) \times \mu C_{i}(z) \\
& O_{i}^{3}=\overline{w_{i}}=\frac{w_{i}}{\sum_{i=1}^{n}} \\
& O_{i}^{4}=\overline{w_{i}} f_{i}=\overline{w_{i}}\left(p_{i} x+q_{i} y+\cdots+r_{i} z+s_{i}\right) \\
& O_{i}^{5}=\sum_{i} \overline{w_{i}} f_{i}=\frac{\sum_{i} w_{i} f_{i}}{\sum_{i} w_{i}}
\end{aligned}
$$


The hybrid learning comprises a forward pass and a backward pass. During the forward pass, the node output goes forward until layer 4 and the consequent parameters are updated by least square method. ${ }^{[14,38]}$ In the backward pass, the error signal propagates backwards and the premise parameters are updated by gradient method. ${ }^{[10]}$ Suppose the PPP training data set has $k$ entries, $U$ represents the matrix of premise parameters, $B$ represents a matrix of consequent parameters and $V$ is a vector of the desired output from the training data set as shown in Equation (17).

Equation (17) can be compressed as follows:

$$
U B=V
$$

where $B$ is an unknown matrix whose elements are from the consequent parameters set. The Least Square Estimator (LSE) of $B, B^{*}$ is given by Equation (19).

$$
B^{*}=\left(U^{T} U\right)^{-1} U^{T} V
$$

where $U^{T}$ is the transpose of $U$ and $U^{-1}$ is the inverse of $U$. The elements of the consequent parameter matrix $B$ are estimated by Equation (19). The LSE $B^{*}$ seeks to minimize the squared error $\|U B-V\|^{2}$ between the computed output and the desired output. ${ }^{[38]}$ The consequent parameters that correspond to this minimum squared error are captured by the DEVS model for detection of activities on PPP.

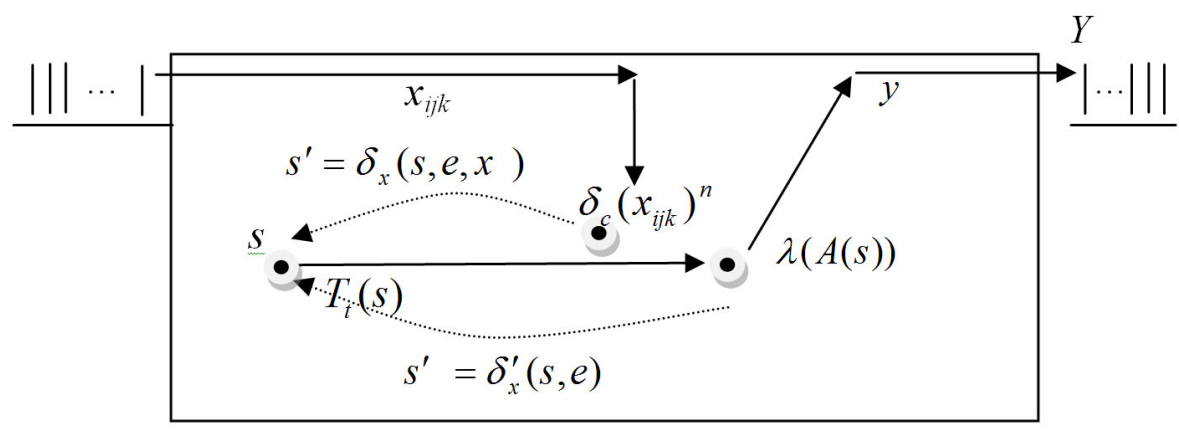

Figure 4. DEVS state transition model

\subsection{DEVS controller}

The DEVS controller for monitoring PPP as illustrated in Figure 4 maintains an internal coupling mechanism which enables the output of one component to be connected to the input of another component.

At any time, the pipeline is in some states. The pipeline continues in this state until there are changes in PPP attributes at time $T_{t}(s)$. PPP data at time $T_{t}(s)$ are ordered by the confluent function $\left(\delta_{c}\right)$ and submitted to the external transition function $\delta_{x}(s, e, x)$ for processing. Time controller converts the time crisp value into a pulse of specific time duration usually a binary code. This binary code is used to activate the discrete event controller to generate specific event for a certain time. The combination of fuzzy logic time control system and discrete event system will form the resultant DEVS. ${ }^{[39]}$ The output function $\lambda$ in the DEVS controller employs the flow rate value to determine the status $y$ of PPP. The DEVS model for PPP monitoring is described in a set form in Equation (20) as follows:

$$
\text { DEVS-ANFIS }=\left\langle X, \delta_{c}, \delta_{x}, T_{t}, \delta_{x}^{\prime}, S, A, \lambda, Y\right\rangle
$$

where $X$ is a set of PPP input variables to be fuzzified; $\delta_{c}: X \rightarrow C$, is the confluent function; $\delta_{x}: X \rightarrow C$, is the external transition function; $T_{t}: S \rightarrow R^{+}{ }_{0, \infty}$, is time assigned for monitoring petroleum pipeline; $\delta_{x}^{\prime}: S \rightarrow S$, is the internal transition function; $S$ is the set of PPP intermediate and final states, Intermediate State $=\{$ Very Low, Low, Moderate, High, Very High $\}$, Final State $=\{$ Normal, Abnormal, Highly Abnormal, Extremely Abnormal $\} ; A: S \rightarrow S$, is the ANFIS function; $\lambda: S \rightarrow Y$, is the output function; $Y$ is a set of PPP status output.

The domain problem model formulation is illustrated thus. Given that $P_{k}, k=1,2, \cdots, q$ is the set of PPP being monitored; $z_{j}, j=1,2, \cdots, m$ represents the set of attributes of PPP; $x_{i}, i=1,2, \cdots, n$ represents the set of records in the PPP dataset collected and $T_{t}, t=R^{+}{ }_{0, \infty}$ represents the discrete time (positive real number between zero and infinity) specified in DEVS model, then the confluent function $\delta_{c}$ which returns the ordered samples of $i$ th data for $j$ th attribute of $k$ th pipeline at time $T_{t}$ is represented as follows:

$$
\delta_{c}\left(x_{i, j, k}, T_{t}\right)=w_{i, j, k}
$$

where $w_{i, j, k}$ is the ordered samples of data $x_{i, j, k}$ at time $T_{t}$. The elements of matrix of ordered attributes of PPP arerepresented by $E=\left\{w_{111}, w_{211}, w_{311}, \cdots, w_{n m q}\right\}$. If $\delta_{x}\left(w_{i, j, k}\right)$ while $\delta_{x}^{\prime}\left(g_{j, k}\right)$ represent external and internal tran- 
sition functions, respectively which return the average of ordered data and the fuzzy value respectively for $j$ th attribute of $k$ th pipeline, then:

$\delta_{x}\left(w_{i, j, k}\right)=\frac{1}{n} \sum_{i=1}^{n} w_{i, j, k} j=1,2, \cdots, m ; k=1,2, \cdots, q$

$$
\delta_{x}^{\prime}\left(g_{j, k}\right)=\left\{\begin{array}{cc}
0 & \text { if } g_{j, k}<a \\
\frac{\left(g_{j, k}-a\right)}{b-a} & \text { if } a \leq g_{j, k}<b \\
\frac{c-\left(g_{j, k}\right)}{c-b} & \text { if } b \leq g_{j, k}<c \\
0 & \text { if } c \leq g_{j, k}
\end{array}\right.
$$

where $g_{j, k}$ is the average of ordered data of PPP; $a, b$ and $c$ represent the parameters of the MF governing triangular shape. The fuzzy value $v_{j, k}$, returned by internal transition function in Equation (23) is used by ANFIS function $A\left(v_{j, k}\right)$ for computation of flow rate as shown in Equation (24).

$$
\begin{gathered}
A\left(v_{j, k}\right)=\sum_{r=1}^{N} \overline{w_{r}} f_{r}=\text { Flow rate }(f) \\
=\left\{\begin{array}{cc}
\text { Very Low } & \text { if } f<0.1 \\
\text { Low } & \text { if } 0.1 \leq f<0.4 \\
\text { Moderate } & \text { if } 0.4 \leq f<0.7 \\
\text { High } & \text { if } 0.7 \leq f<0.8 \\
\text { Very High } & \text { if } 0.8 \leq f<1.0
\end{array}\right.
\end{gathered}
$$

where $r=1,2 \cdots, N$ represents rules that govern the input and output events on PPP based on Sugeno inference mechanism. $\overline{w_{r}}$ is the normalized firing strength of rules $f_{r}$ represents $\mathrm{CMF}$ which determines the flow rate.

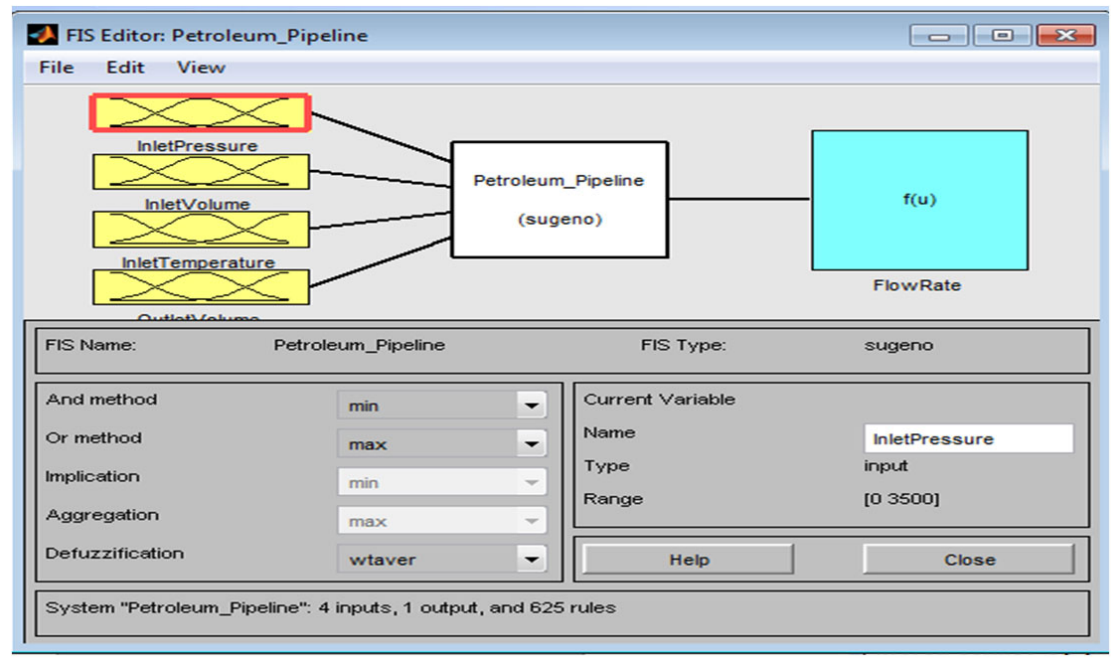

Figure 5. ANFIS inference editor

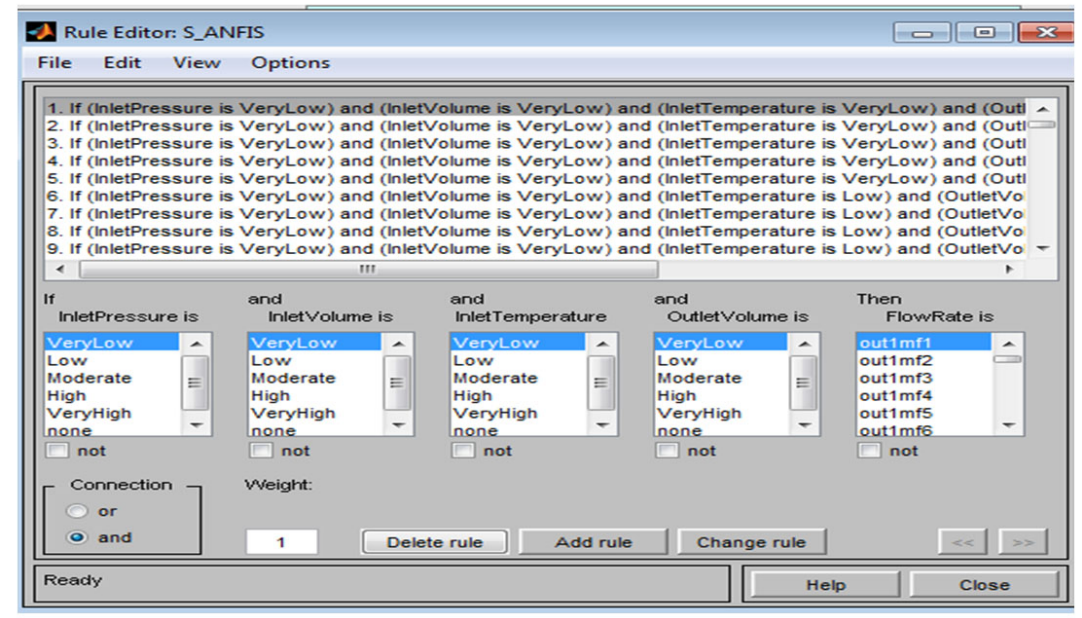

Figure 6. ANFIS rule base editor 


\section{IMPLEMENTATION AND DISCUSSION}

The system was implemented with NetBeans IDE 7.3.1 and MatLab 7.10 (R2010a) as the front-end tools; Microsoft Excel and MYSQL Wamp Server 2.2 were the database management tools.

Matlab 7.10 (2010a) was chosen because of the rich libraries of machine learning tools and the ease of defining FL function blocks and implementing FL and ANFIS as well as DEVS. The other tools are compatible with Matlab 2010a and provided support for integrated processing of PPP parameters. ANFIS Inference and Rule Base Editors are shown in Figures 5 and 6 respectively. The rulebase has a total of 625 rules; this is evaluated from five (5) membership functions and four (4) attributes of PPP.

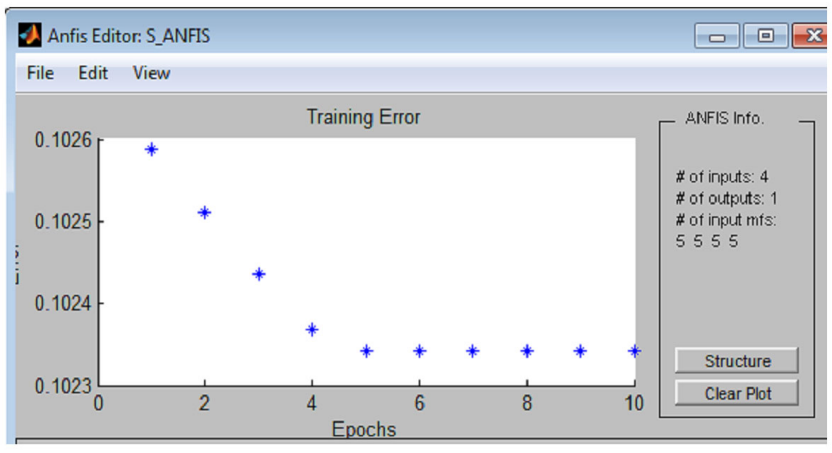

Figure 7. ANFIS training with hybrid algorithm

\subsection{ANFIS training procedure}

The ANFIS training window using hybrid learning algorithm is depicted in Figures 7 and 8. It accepts four (4) input variables and one (1) output variable. Each of these variables has five (5) membership functions, namely: Very Low, Low, Moderate, High and Very High. The optimization method employed is hybrid algorithm with error tolerance of 0.001 . The result shows that training error decreases as epoch increases (from 1 to 5) and maintains a constant value from epoch 6 with average training error of 0.10234 . The testing error at epoch 10 is depicted in Figure 7, while the summary of training, testing, checking and average errors from epoch 1 to 10 are presented in Table 2 for hybrid algorithm and Table 3 for back propagation algorithm.

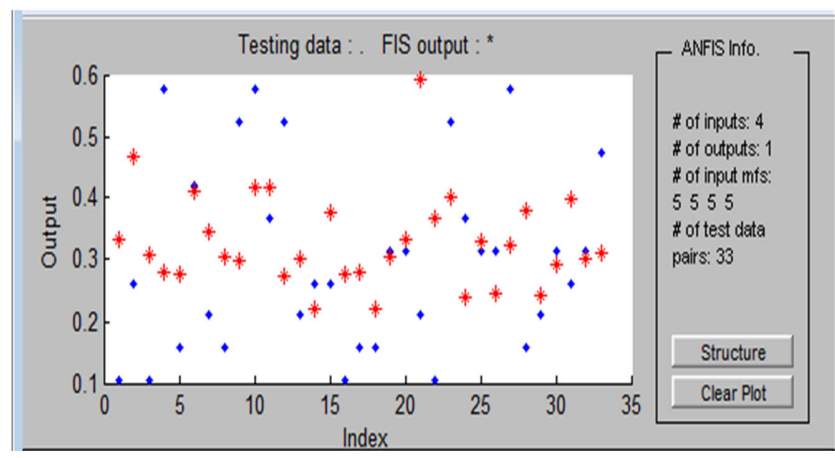

Figure 8. Plot of FIS input and ouput data

Table 2. ANFIS performance with hybrid algorithm

\begin{tabular}{llllll}
\hline SN & Number of epochs & Training error & Checking error & Testing error & Average error \\
\hline 1 & 100 & 0.10259 & 0.12960 & 0.12949 & 0.12056 \\
2 & 200 & 0.10251 & 0.12957 & 0.12948 & 0.12052 \\
3 & 300 & 0.10244 & 0.12945 & 0.11946 & 0.11712 \\
4 & 400 & 0.10237 & 0.12943 & 0.11945 & 0.11708 \\
5 & 500 & 0.11980 & 0.11920 & 0.01023 & 0.08307 \\
6 & 600 & 0.10234 & 0.17394 & 0.16512 & 0.14713 \\
7 & 700 & 0.10234 & 0.17394 & 0.16512 & 0.14713 \\
8 & 800 & 0.10234 & 0.17394 & 0.16512 & 0.14713 \\
9 & 900 & 0.10234 & 0.17394 & 0.16512 & 0.14713 \\
10 & 1000 & 0.10234 & 0.17394 & 0.16512 & 0.14713 \\
\hline
\end{tabular}

A comparison of hybrid learning with back propagation algorithm shows that ANFIS hybrid learning is relatively faster to implement than back propagation algorithm. Hybrid learning algorithm requires a few number of training epochs to converge, for instance in Table 3 the least training error is observed at epoch 5 . This training epoch is relatively small compared to the 500 training epochs which has the least average error of 0.1203 in ANFIS back propagation algorithm performance evaluation of Table 3 . The quick convergence Published by Sciedu Press and fast reduction in training errors observed in the hybrid learning algorithm is due to the integration of least square strategy in the forward phase and back propagation gradient descent method in the backward phase. In this work, hybrid learning strategy is employed and the values of consequent paramaters with least testing and checking errors are extracted and used in the DEVS system for classification of activities on PPP. 
Table 3. ANFIS performance with backpropagation algorithm

\begin{tabular}{llllll}
\hline SN & Number of epochs & Training error & Checking error & Testing error & Average error \\
\hline 1 & 100 & 0.1383 & 0.1542 & 0.1513 & 0.1479 \\
2 & 200 & 0.1295 & 0.1460 & 0.1442 & 0.1399 \\
3 & 300 & 0.1208 & 0.1378 & 0.1388 & 0.1325 \\
4 & 400 & 0.1120 & 0.1296 & 0.1325 & 0.1247 \\
5 & 500 & 0.1103 & 0.1214 & 0.1292 & 0.1203 \\
6 & 600 & 0.1102 & 0.1533 & 0.1751 & 0.1462 \\
7 & 700 & 0.1101 & 0.1751 & 0.1868 & 0.1573 \\
8 & 800 & 0.1200 & 0.1869 & 0.1831 & 0.1633 \\
9 & 900 & 0.1602 & 0.1982 & 0.1934 & 0.1839 \\
10 & 1000 & 0.1664 & 0.2180 & 0.1992 & 0.1945 \\
\hline
\end{tabular}

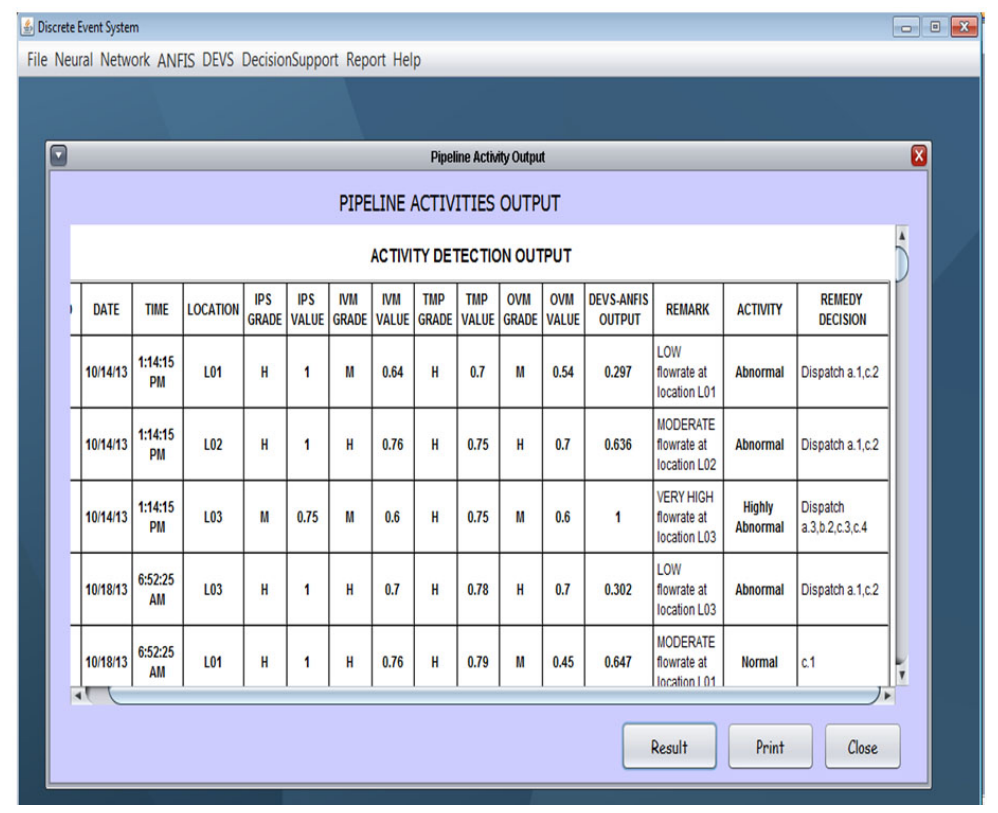

Figure 9. PPP events classification using DEVS-ANFIS

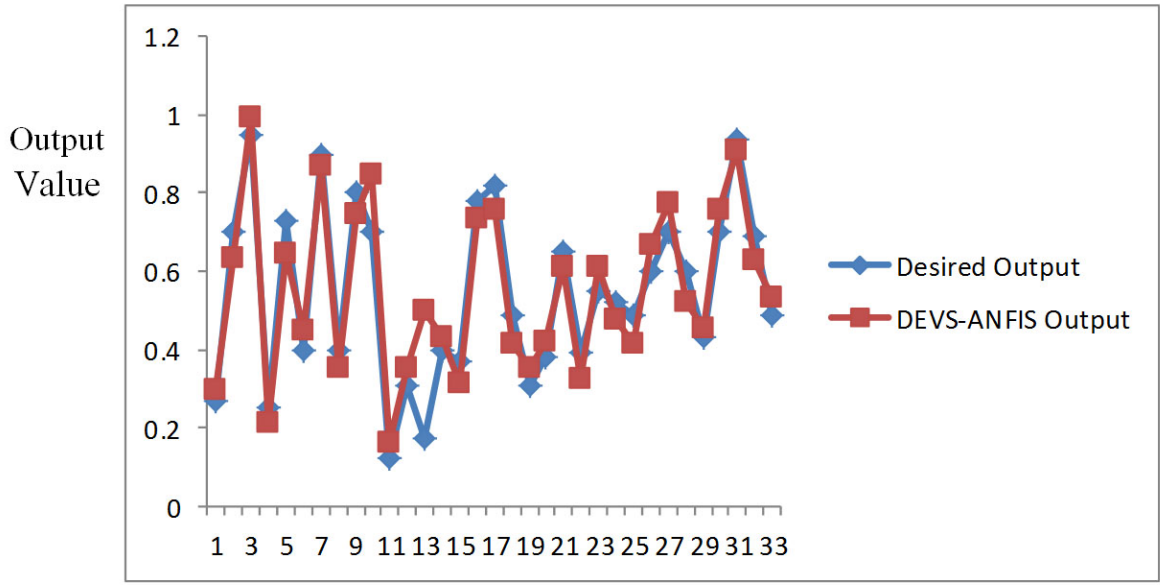

Test Data Series

Figure 10. DEVS-ANFIS and desired output correlation 


\subsection{DEVS-controller Results}

DEVS automatically monitors the input interface using the time and event contollers, detects presence of new data and coordinates for the PPP data computation while ANFIS provides the intellegence needed for efficient evaluation of PPP status. Figures 9 and 10 show PPP events classification using DEVS-ANFIS and comparison with desired output respectively. As shown in Figure 10, the desired output value of 0.27 and DEVS-ANFIS value of 0.297 are observed for test data series 1 . The desired and DEVS-ANFIS outputs increase to 0.643 and 0.636 respectively at test data 2 . The values of 0.98 and 1.000 are observed for desired and DEVSANFIS outputs at test data 3, these values drop to 0.24 and 0.210 respectively at test data 4 .

The value of desired and DEVS-ANFIS outputs tend to each other at every test data series with exception of test data 13 where the desired output value of 0.17 differs significantly from DEVS-ANFIS output of 0.521 . Out of 33 data samples in test data series, 32 correct detections and 1 incorrect detection were observed. The Mean Squared Error (MSE) value of 0.0303 and correlation coefficient (r) value of 0.9697 was obtained between the desired and the computed output. This gives $96.97 \%$ correct detections on the test data.

\section{Conclusions}

This paper presents the development of a DEVS-ANFIS platform suitable for monitoring PPP with automatic tools for timing and dealing with simultaneous events from multi pipeline locations as well as proposed an in-built intelligent deductive inference mechanism for PPP status evaluation and promotion of timely response to pipeline anomalies in the petroleum industry. The introduction of cognitive reasoning and self adaptive capabilities into DEVS systems enhance the real time control and monitoring of these dynamic events.
It provides a speedy classification with minimized error level, 96.97\% accuracy. The performance of DEVS-ANFIS on the monitoring of pipeline activities yielded correlation coefficient (r) and Mean Squared Error (MSE) of 0.9697, 0.0303 respectively. These results are significantly better than performance of Support Vector Machine (SVM) and Extreme Learning Machine (ELM) of 0.1508 and 0.4258 for SVM and 0.1017 and 0.2063 for ELM Normalized Root Mean Square Error (NRMSE) in the prediction of magnitude of leaks and location of leaks reported in Salam et al. ${ }^{[40]}$ DEVS-ANFIS is superior to SVM and ELM tools, therefore more suitable for classification and detection of events on PPP.

This research, indeed develops an intelligent hybrid system driven by DEVS and ANFIS for classification of activities on PPP. In the ANFIS model, hybrid learning algorithm was observed to converge faster than the back propagtion algorithm in the detection of flowrate of petroleum products in pipeline. DEVS coordinates PPP input and its transition functionsto classify activities on PPP. A testing error of 0.0303 was observed in the evaluation of DEVS-ANFIS system on 33 test data set, 32 precise detections were observed with 1 incorrect detection. The DEVS-ANFIS system could resolve conflicts of random data transmitted to the base station from various petroleum products pipelines and could monitor, detect, localize, quantify and classify activities such as "Very Low", "Low", "Moderate", "High", "Very High" from multilocations PPPwith $96.97 \%$ level of confidence. As futher directions, visualization of the underlying structures and relationships between the time and control parameters of PPP in an unsupervised marnner will be demonstrated. In addition, design and development of techniques to formally interface DEVS-ANFIS towards the creation of interactive virtual reality and simulation-based platform for PPP monitoring is necessary.

\section{REFERENCES}

[1] Ogwu FA. Challenges of Oil and Gas Pipeline Network and the role of Physical Planners in Nigeria. FORUM Ejournal. 2011 June; 10: 41-51.

[2] Achebe C, Nneke UC, Anisiji OE. Analysis of Oil Pipeline Failures in the Oil and Gas Industries in the Niger Delta Area of Nigeria. In proceeding of the International MultiConference of Engineers and Computer Sceintists IMEC. Hong Kong. 2012 March: 14-6.

[3] Wilson RA. Transportation in America, Eighteenth Edition. Washington, D.C.: Eno Transportation Foundation, Inc.; 2001

[4] Trench CJ. How Pipelines Make the Oil Market Work - Their Networks, Operation and Regulation. A Memorandum Prepared for the Association of Oil Pipe Lines And the American Petroleum Institute's Pipeline Committee. Allegro AllegroAllegro EnergyGroup; 2001. Accessed 8th June, 2016. http: //www . iatp.org/files/4 51_2_31375.pdf

Published by Sciedu Press
[5] Okoli AC, Orinya S. Oil Pipeline Vandalism and Nigeria's National Security. Global Journal of Human Social Science Political Science. 2013; 13(5): 1-10.

[6] Ogbeni OO. Fuel Pipeline Vandalism in Nigeria; 2012 http://wwwchatafrik.com/articles/economy/item /1287-Fuel-Pipeline-Vandalism-in-Nigeria.html Accessed December 20, 2012.

[7] Vishash S. 2013 Vibro-Acoustic Monitoring of Pipeline Leakage and Corrosion.M.Sc. Thesis, Department of Mechanical and Manufacturing Engineering, University of Calgary, Algerta. 2013: 47-105.

[8] Niklès M, Ravet F, Briffod FA. Comprehensive Long Distance and Real- Time Pipeline Monitoring System Based on Fiber Optic Sensing. Brazilian Petroleum, Gas and Biofuels Institute - IBP, Technical Paper in the Rio Pipeline Conference Proceedings. 2009: 56-60.

[9] Daniel D, Mihaela OA. Fuzzy Logic Based System for Heavy Metals Load Waste Water Monitoring. Proceedings of 4th International 
Symposium on Intelligence. October 23-25, 2009. Huangshi, China.

[10] Udoh SS. Artificial Neural Network for the Prediction of the Demand of Petrol at the Depot. An M. Tech Thesis, Department of Computer Science, Federal University of Technology, Akure; 2009.

[11] Santos RB, de Sousa EO, da Silva FV, et al. Detection and on-line Prediction of Leak Magnitude in a Gas Pipeline using an Acoustic Method and Neural Network Data Processing. Brazilian Journal of Chemical Engineering. 2013; 13(1): 60-7.

[12] Akinyokun OC, Inyang UG. Experimental Study of Neuro-FuzzyGenetic Framework for Oil Spillage Risk Management. Artificial Intelligence Research. 2013; 2(4): 14-36. https ://doi.org/10.5 430/air.v2n4p13

[13] Adewuyi PA, Okelola MO. An Investigation into the Use of Artificial Neural Network to Monitor and Detect Leakage Point(s) along a Select Pipeline. Pacific Journal of Science and Technology. 2013; 14(1):96-101.

[14] Inyang UG, Akinyokun OC. A Hybrid Knowledge Discovery System for Oil Spillage Risks Pattern Classification. Artificial Intelligence Research. 2014; 3(4): 73-86. https://doi.org/10.5430/air v3n $4 \mathrm{p} 77$

[15] Inyang UG. Development of Knowledge Discovery System for Oil Spillage Risk Management. Ph.D. Dissertation, Department of Computer Science, Federal University of Technology, Akure; 2012.

[16] Zeigler BP. DEVS Today: Recent Advances in Discrete Event-Based Information Technology", Proceedings of the 11th IEEE/ACM International Symposium on Modeling, Analysis and Simulation of Computer Telecommunications Systems, Orlando, FL. 2003: 148-61. https ://doi.org/10.1109/mascot.2003.1240652

[17] Mohammad M. Real-Time and Embedded Systems Development based on Discrete Event Modeling and Simulation. PhD Thesis Dissertation, Carleton University Ottawa, Ontario, Canada. 2011: 56-84.

[18] Lewis N, Xiaolin H. DEVS-Fire: Towards an Integrated Simulation Environment for Surface Wildfire Spread and Containment. Department of Industrial and Systems Engineering. Texas University, USA. International Journal for Modeling and Simulation. 2008; 8(4): 13755.

[19] Prasanna S, Shahab S, Shan X, et al. Multi Agent Simulation using Discrete Event and Soft-computing Methodologies. IEEE Journal of Simulations. 2008; 4(2): 1711-6.

[20] Eduardo P, Lewis N, Eunshin B, et al. A Stochastic DEVS Wind Turbine Component Model for Wind Farm Simulation. Department of Industrial and systems Engineering, Texas University Journal. 2011 4(2): 34-56.

[21] Mariano Z, Rodrigo C, Gabriel W, et al. Architecture for Integrated Modelling, Simulation and Visualisation of Environmental System using GIS and Cell-DEVS.Proceedings of the 2011 Winter Simulation Conference. December 11-14, 2011, Phoenix, USA.

[22] Udoh SS. Adaptive Neuro-Fuzzy Discrete Event System Specification for Monitoring Petroleum Products Pipeline. Ph.D. Dissertation, Department of Computer Science, Federal University of Technology, Akure, Nigeria. 2016: 30-156.

[23] Zeigler B. Theory of Modeling and Simulation. Wiley, N. Y.; 1976.

[24] Shang H,Wainer GA. A flexible dynamic structure DEVS algorithm. Towards embedded systems. Proceedings of the Summer Computer Simulation Conference. 2006: 339-45.
[25] Cao Y, Ying M, Chen G. State-Based Control of Fuzzy Discrete Event Systems IEEE Transactions on Systems, Man, and Cybernetics; 2006.

[26] Filippi J, Bisgambiglia P. JDEVS: An Implimentation of a DEVS Based Formal Framework for Environment Modelling. Elsevier, Environmental Modelling and Software. 2004; 19(2): 261-74. https : //doi.org/10.1016/j.envsoft.2003.08.016

[27] Ulrich AKT. Application of an Oil Spill Response Model in IceBridge Simulator Training. Masters Thesis. Department of Marine Technology, Norwegian University of Science and Technology. 2011: 67-89.

[28] Lin F, Ying H. Fuzzy discrete event systems and their observability. in Proc. Joint Int. Conf. 9th Int. Fuzzy Systems Assoc. World Congr. 20th North America Fuzzy Information. Processing Society, Vancouver, BC, Canada. 2001 July: 1271-6.

[29] Santucci J, Capocchi L. Fuzzy Discrete-Event Systems Modeling and Simulation with Fuzzy Control Language and DEVS Formalism. The Sixth International Conference on Advances in System Simulation. 2014: 250-5.

[30] Sheikh-Bahaei S, LIU J, Jamshidi MO. An Intelligent Discrete Event Approach To Modeling, Simulation And Control Of Autonomous Agents. Intelligent Automation and Soft Computing. 2004; 10(4): 337-48. https : //doi.org/10.1080/10798587.2004.10 642887

[31] Goran S, Stojan P, Mitja RK, et al. Evacuation Model Managed through Fuzzy Logic during an Accident in a LNG Terminal. Scientific Journal. 2013; 36(108): 131-7.

[32] Mashford J, De S, Marney D, et al. An Approach to Leak Detection in Pipe Networks Using Analysis of Monitored Pressure Values by Support Vector Machine.International Journal of Network and Security Systems. 2009; 4(5): 534-9. https ://doi.org/10.1109/ns s. 2009.38

[33] Zeigler B. Multifaceted Modeling and discrete event simulation. Academic Press, Boston; 1984.

[34] Ziegler B. DEVS: Past, Present and Future. Journal of DEVS, Cagese, Corsica. 2016: 1-17.

[35] Chow AC, Zeigler BP. Parallel DEVS: A Parallel, Hierarchical, Modular Modeling Formalism. Proceedings of Winter Simulation Conference. 1994: 716-22. https://doi.org/10.1109/WSC.1994.717 419

[36] Youcef M, Maamar A. Specificaton of the Sate's Lifetime in the DEVS Formalization by formalism by Fuzzy Controller. International Journal of Advanced Information Technology (IJAIT). 2013; 3(2): 1-5. https://doi.org/10.5121/ijait. 2013.3201

[37] Jamshidi M, Zilouchian A. Intelligent Control Systems using Soft Computing Methodologies. CRC Press, Boca Raton, FL; 2001.

[38] Loganathan C, Girjia KV. Investigation on Hybrid Learning in ANFIS. International Journal of Engineering Research and Applications. 2014; 4(10): 31-7.

[39] Kwon YH, Park SJ, Kim T. Fuzzy-DEVS formalism: Concepts, Realization and Applications. In AI, Simulation and Planningin High Autonomy Systems. 1996, EPDUniversity of Arizona, San Diego.

[40] Salam A, Tola M, Selintung M, et al. Application of SVM and ELM Methods to Predict Location and Magnitude Leakage of Pipelines on Water Distribution Network. International Journal of Advanced Computer Research. 2015; 5(19): 139-44. 